\title{
Friction and Wear Measurements with an Adapted Ball-On-Pyramid Test Setup
}

\author{
Viktor Krasmik* and Josef Schlattmann \\ Workgroup on System Technologies and Engineering Design Methodology, \\ Hamburg University of Technology \\ Denickestrasse 17, 21073 Hamburg, Germany \\ *Corresponding author: viktor.krasmik@tuhh.de
}

( Manuscript received 26 August 2015; accepted 30 December 2015; published 30 April 2016 )

( Presented at the International Tribology Conference Tokyo 2015, 16-20 September, 2015 )

\begin{abstract}
In tribological testing with simplified test geometries, the contact configurations usually consist of two bodies representing one material pairing. This contribution introduces an adapted ball-on-pyramid test configuration which allows the experimental investigation of combined contact configurations consisting of more than one material pairing. In the proposed test setup, a ball is loaded against four flat test samples positioned in a pyramid-like sample holder with an opening angle of $90^{\circ}$. The test samples are arranged on the circumference in a regular manner. The ball rotates uniformly around its vertical axis and slides over the samples. The coefficient of friction, the running-in wear, the linear wear rate, the wear scar diameter, and the weight loss of four different sample materials and their combinations are compared and discussed. The obtained results for combined contact configurations with more than one material pairing show that a manipulation of the friction and wear characteristics is possible.
\end{abstract}

Keywords: friction, wear, tribological testing, ball-on-pyramid test setup, material combination

\section{Introduction}

Generally, a tribological system consists of a main body, an opposing body, an intermediate material, and a surrounding medium. In order to improve or manipulate the friction and wear performance of a tribological system, different strategies exist. Common approaches are the use of improved materials with regard to the desired properties, the employment of lubrication, or the application of coatings [1].

In a first step, usually simplified test setups and geometries are used for characterizing the tribological behavior of material pairings. For that purpose, many test setups with different contact configurations (pin-on-disc, flat-on-flat, block-on-ring, four-ball test, etc.) exist [2].

A rough classification of the test configurations can be made regarding the number of bodies in contact, respectively the number of samples. Contact configurations like pin-on-disk or flat-on-flat usually consist of two bodies, whereas configurations like the four-ball test consist of more than two bodies.

In the four-ball test, a rotating ball is loaded against three static balls. The test is often used, for example, to assess the performance of lubricating fluids with respect to wear prevention according to ASTM D4172-94 [3] or to determine the coefficient of friction according to ASTM D5183-08 [4].

On the basis of the four-ball test and primarily for evaluating the fuel injection pump wear, many more related test geometries have been developed.

In the ball-on-three-seats (BOTS) setup, a rotating ball is pressed against three obliquely positioned conforming seats. Due to the larger area, the contact pressures are lower. The wear test results are very sensitive to manufacturing tolerances of the conforming seats, making the test configuration less reliable and practical [5]. Therefore, a ball-on-three-disks (BOTD) setup, representing a modified version of the BOTS setup but with a simpler sample geometry and less tolerance restrictions, has been proposed by Voitik and Ren [6] and used for characterizing fuel lubricity.

Fildes et al. [7,8] introduced an improved ball crater micro-abrasion test method based on the BOTD configuration. The method was used to demonstrate the performance benefits of many modern coatings and the effect of different coating design strategies on abrasive and adhesive wear. Furthermore, the advantages of the method in comparison to conventional test methods were highlighted.

A similar test setup consisting of a rotating cone, which is pressed against three cylinders/rollers, is defined in the PN-83/H-04302 [9] standard and intended 
for friction and wear measurements. In order to characterize the wear resistance of surface hardened materials, Senatorski et al. [10,11] used the threecylinders-cone, respectively three-rollers-cone wear test and compared it to widely used tests such as the pin-and-vee block method or the disk-on-disk method. Based on the obtained results, it is claimed that the proposed setup is more appropriate for testing surface hardened materials.

A simplified version of the BOTD configuration is the ball-on-prism setup. According to ISO 7148-2 [12], the configuration is intended for evaluating polymerbased plain bearing materials. The test geometry, consisting of a ball in contact with two obliquely positioned flat samples, reproduces the contact conditions in a bearing bushing, where the contact pressure increases while the contact area decreases with the run time.

Krasmik and Schlattmann [13] used an adapted ball-on-prism test setup with two, three, and four flat samples (all samples of identical material) to study the friction and wear behavior of dry metal-metal pairings. The influence of the normal load, the sliding velocity, and the number of contact points, respectively test samples were investigated and discussed. In that study, no noticeable dependency between the coefficient of friction and the number of contact points, respectively number of flat samples was observed. But an increasing trend for the wear rate as a function of the number of contact points was evident.

In general, such configurations provide simple and reproducible contact geometries with a wide range of test possibilities under various conditions. Friction and wear measurements under dry or lubricated (oil, grease, or powder) conditions are possible.

So far, such test setups were used for testing contact configurations where the main body and the opposing body are of the same or different materials, in both cases representing a single material pairing. In contrast to that, an adapted ball-on-pyramid test setup is proposed in this paper with which samples of different materials can be tested at the same time, thus allowing the experimental investigation of combined contact configurations consisting of more than one material pairing. The aim of the study is to introduce an appropriate test procedure and to investigate whether and to what extent manipulations of the friction and wear performance by such a combined contact configuration are generally possible. This contribution represents some fundamental research without focusing on certain specific applications at this early stage. For that purpose, some exemplary metal-metal pairings are used and the effect on the friction and wear characteristics is discussed.

\section{Experimental method}

\subsection{Ball-on-pyramid contact configuration}

The proposed ball-on-pyramid contact setup is
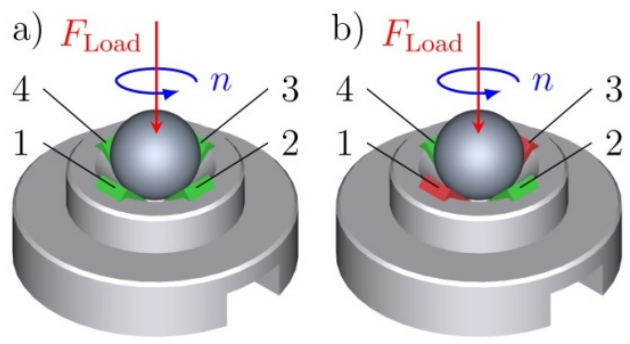

Fig. 1 Ball-on-pyramid sample holder with four samples: a) basic contact configuration, b) combined contact configuration

illustrated in Figure 1. Four flat test samples are arranged in a pyramid-like sample holder with an opening angle of $90^{\circ}$ in a regular manner. A ball, which is uniformly rotating around its vertical axis, is pressed against the flat samples and slides over the surfaces of the samples.

Contact configurations with all four samples being of the same material as well as configurations consisting of two different sample materials are possible. Due to a slot and key principle, the sample holder can adapt in the horizontal plane to achieve a uniform load distribution.

Determining whether a balanced or unbalanced load distribution is present during the test is a complex task. So far, there is no appropriate criterion in literature for that special case. Measuring the local temperature with thermocouples or the stresses with strain gauges of the flat samples near the contact points during the test could provide more insight. A more straightforward approach is to compare the resulting wear marks of samples positioned opposite each other after the test. In this study, the latter approach was chosen. So, if the resulting diameters of samples positioned opposite each other deviated by more than $20 \%$, it was considered as an indication for an unbalanced load distribution and was therefore not used for evaluation. Comparing the different standard deviations of the obtained results showed that $20 \%$ are a reasonable limit in order to have a standard deviation for the resulting wear scar diameter (not more than $10 \%$ of the mean value) which is in the range of the other wear characteristics (such as the running-in wear, the linear wear rate, and the weight loss).

A schematic representation of the contact condition in a ball-on-pyramid setup is depicted in Figure 2.

The sliding velocity at the contact points is

$$
v=2 \pi n \frac{D}{2} \cos (\alpha)=14.1 \mathrm{~mm} / \mathrm{s},
$$

where $n=30 \mathrm{rpm}$ is the rotational speed, $D=12.7 \mathrm{~mm}$ the diameter of the rotating ball, and $\alpha=45^{\circ}$ the half opening angle.

The ball is loaded against the flat samples with a defined load of $F_{\text {Load }}=100 \mathrm{~N}$. The total resulting normal load is 


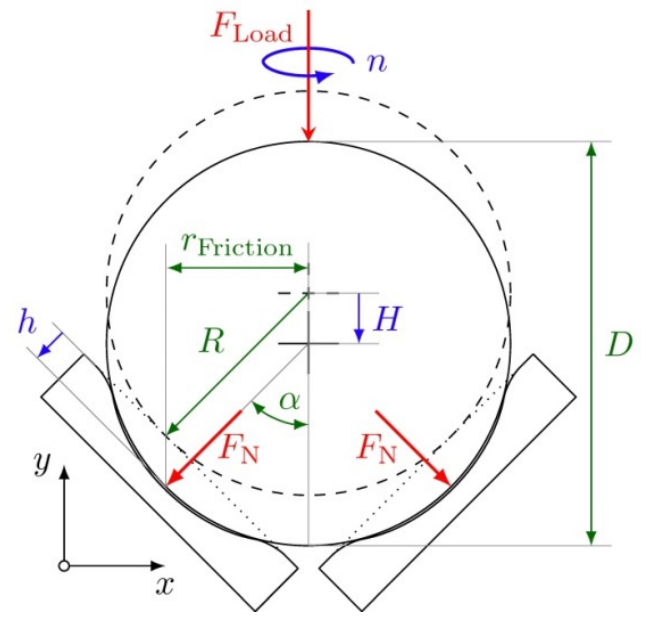

Fig. 2 Schematic representation of the contact condition in a ball-on-pyramid setup

$$
F_{\text {N,total }}=\frac{F_{\text {Load }}}{\cos (\alpha)}=141.4 \mathrm{~N} \text {. }
$$

For samples of the same material, the resulting normal load per sample is

$$
F_{\mathrm{N}}=\frac{F_{\text {Load }}}{n_{\text {Contact }} \cos (\alpha)}=35.4 \mathrm{~N},
$$

where $n_{\text {Contact }}=4$ is the number of contact points, respectively test samples.

\subsection{Materials and preparation}

Flat samples with the dimensions of $8 \mathrm{~mm} \times 5 \mathrm{~mm} \times$ $1 \mathrm{~mm}$ were prepared from commercially available brass (CuZn37), copper (SF-Cu), aluminum (AlMg3), and steel (St-37) sheets. Bearing steel balls (100Cr6) were used as the rotating counterpart.

$\mathrm{CuZn} 37$ is a copper-zinc alloy having a homogeneous alpha phase structure and a small amount of beta phase. SF-Cu crystallizes in a cubic face-centered lattice structure with some twin formations. AlMg3 is an alloy from the wrought aluminum-magnesium group. St-37 is an unalloyed construction steel with a microstructure consisting of ferrite and lamellar pearlite. The bearing steel is a fine-grained low alloy martensitic chrome steel, which is hardened by heat treatment up to the core.

These materials were chosen since they have different mechanical and tribological properties. Furthermore, such materials and their related alloys are often used in many industrial applications. The main reasons for that are their thermal and electrical characteristics, their wear and friction performance, their corrosion resistance, and their comparatively low costs. Some mechanical properties of the used materials and the corresponding material numbers are listed in Table 1.

Every test was conducted with new bearing steel balls and new flat test samples. The samples were cleaned with a technical cleaner (isoparaffin based solvent cleaner) in an ultrasonic bath and dried with compressed air before the tests.

Four basic contact configurations and six combined contact configurations were studied. In this context, the basic contact configurations represent the contact between four samples of the same material and the rotating bearing steel ball and the combined contact configurations the contact between four samples of two different materials and the rotating bearing steel ball (see Figure 1). In the case of combined contact configurations, samples of the same material were arranged opposite each other. A summary of the investigated contact, respectively material configurations is given in Table 2.

\subsection{Theoretical consideration}

For a combined contact configuration (samples are not of the same material), the applied load is distributed according to the elastic properties of the corresponding materials. In order to estimate the resulting normal load for that case, the assumption is made that all four samples experience the same indentation depth of the bearing steel ball and that there is no wear present. Applying Hertz theory for the contact of a sphere with an elastic half-space leads to the following relation between the normal load per sample $F_{\mathrm{N}, i}$ and the indentation depth $d$ [14]:

Table 1 Properties of the used test materials

\begin{tabular}{c|c|c|c|c} 
Material & Hardness [HRV] & $\boldsymbol{R}_{\mathbf{a}}[\boldsymbol{\mu} \mathbf{m}]$ & Young's modulus [GPa] & Poisson's ratio [-] \\
\hline AlMg3 (3.3535) & 77 & $<0.4$ & 70 & 0.3 \\
CuZn37 (2.0321) & 131 & $<0.4$ & 110 & 0.3 \\
SF-Cu (2.0090) & 80 & $<0.4$ & 130 & 0.3 \\
St-37 (1.0110) & 114 & $<0.4$ & 210 & 0.3 \\
100Cr6 (1.3505) & $700-860$ & $<0.03$ & 210 & 0.3
\end{tabular}


Table 2 Investigated basic and combined contact configurations

\begin{tabular}{|c|c|c|c|}
\hline Notation & $\begin{array}{c}\text { Contact } \\
\text { configuration }\end{array}$ & $\begin{array}{l}\text { Material pairings per } \\
\text { contact configuration }\end{array}$ & $\begin{array}{c}\text { Max. contact pressure } \\
\text { per sample [MPa] }\end{array}$ \\
\hline 4A-BS & $4 \times \operatorname{AlMg} 3-100 \mathrm{Cr} 6$ & 1 & 8.27 \\
\hline 4B-BS & $4 \times \mathrm{CuZn} 37-100 \mathrm{Cr} 6$ & 1 & 10.22 \\
\hline 4C-BS & $4 \times$ SF-Cu $-100 \mathrm{Cr} 6$ & 1 & 10.97 \\
\hline 4S-BS & $4 \times$ St- $37-100 \mathrm{Cr} 6$ & 1 & 13.13 \\
\hline $2 \mathrm{~A} / 2 \mathrm{~B}-\mathrm{BS}$ & $2 \times \mathrm{AlMg} 3 / 2 \times \mathrm{CuZn} 37-100 \mathrm{Cr} 6$ & 2 & $7.81(-5.6 \%) / 10.73(+5 \%)$ \\
\hline $2 \mathrm{~A} / 2 \mathrm{C}-\mathrm{BS}$ & $2 \times \mathrm{AlMg} 3 / 2 \times \mathrm{SF}-\mathrm{Cu}-100 \mathrm{Cr} 6$ & 2 & $7.64(-7.6 \%) / 11.69(+6.6 \%)$ \\
\hline $2 \mathrm{~A} / 2 \mathrm{~S}-\mathrm{BS}$ & $2 \times \mathrm{AlMg} 3 / 2 \times \mathrm{St}-37-100 \mathrm{Cr} 6$ & 2 & $7.22(-12.7 \%) / 14.44(+10 \%)$ \\
\hline 2B/2S-BS & $2 \times \mathrm{CuZn} 37 / 2 \times \mathrm{St}-37-100 \mathrm{Cr} 6$ & 2 & $9.55(-6.6 \%) / 13.89(+5.8 \%)$ \\
\hline $2 \mathrm{C} / 2 \mathrm{~S}-\mathrm{BS}$ & $2 \times \mathrm{SF}-\mathrm{Cu} / 2 \times \mathrm{St}-37-100 \mathrm{Cr} 6$ & 2 & $10.46(-4.6 \%) / 13.68(+4.2 \%)$ \\
\hline $2 \mathrm{~B} / 2 \mathrm{C}-\mathrm{BS}$ & $2 \times \mathrm{CuZn} 37 / 2 \times \mathrm{SF}-\mathrm{Cu}-100 \mathrm{Cr} 6$ & 2 & $10.04(-1.8 \%) / 11.16(+1.7 \%)$ \\
\hline
\end{tabular}

$F_{\mathrm{N}, i}=\frac{4}{3} E_{i}^{*} \sqrt{R} d^{3 / 2}$,

$\frac{1}{E_{i}^{*}}=\frac{1-v_{1}^{2}}{E_{1}}+\frac{1-v_{2}^{2}}{E_{2}}$,

with $E_{i}^{*}$ being the effective elastic modulus ( $E_{1}$ and $E_{2}$ are the Young's moduli of the contacting bodies and $v_{1}$ and $v_{2}$ are their Poisson's ratios) and $R$ the radius of the bearing steel ball.

Furthermore, a symmetric load distribution is assumed, meaning that the opposing samples experience the same loads and that the contact configuration is balanced.

Taking all these assumptions and relations into account leads to the following relation between the normal loads per sample ( $X$ and $Y$ indicate the corresponding material combination):

$$
F_{\mathrm{N}, 2 X}=\frac{E_{2 X}^{*}}{E_{2 Y}^{*}} F_{\mathrm{N}, 2 Y} \cdot
$$

From the balance of forces, a relation between the applied load and the normal loads can be derived:

$$
\begin{aligned}
& F_{\text {Load }}=\frac{n_{\text {Contact }}}{2} F_{\mathrm{N}, 2 X} \cos (\alpha)+\frac{n_{\text {Contact }}}{2} F_{\mathrm{N}, 2 Y} \cos (\alpha), \\
& F_{\mathrm{N}, 2 X}=\frac{F_{\text {Load }}}{\frac{n_{\text {Contact }}}{2} \cos (\alpha)}\left(\frac{E_{2 X}^{*}}{E_{2 X}^{*}+E_{2 Y}^{*}}\right), \\
& F_{\mathrm{N}, 2 Y}=\frac{F_{\text {Load }}}{\frac{n_{\text {Contact }}}{2} \cos (\alpha)}\left(\frac{E_{2 Y}^{*}}{E_{2 X}^{*}+E_{2 Y}^{*}}\right),
\end{aligned}
$$

For samples of the same material, Eq. (8) and Eq. (9) reduce to Eq. (3).

With these normal loads, the maximum contact pressure per sample can be calculated [14]:

$$
p_{0,2 i}=\frac{1}{\pi}\left(\frac{6 F_{\mathrm{N}, 2 i} E_{2 i}^{* 2}}{R^{2}}\right)^{1 / 3} \text {. }
$$

In Table 2, the resulting maximum contact pressures per sample are listed. The percentage changes of the contact pressures of the combined configurations with respect to the pressures of the basic configurations are also indicated. Furthermore, the maximum contact pressures per sample are illustrated in Figure 3 for a better comparison. For the calculation of the contact pressures, the corresponding material properties, which are listed in Table 1, were used.

The results show that by combining samples of different materials, the contact pressure distribution per sample is affected. While for the basic contact configurations an equal load distribution per sample can be expected, this is not the case for combined contact configurations. The resulting contact pressures per sample depend on the properties of the corresponding materials. The contact pressures increase for samples with higher Young's moduli and decrease for samples with lower Young's moduli. Due to the elastic properties, the largest relative change of contact pressure per sample can be observed for the $2 \mathrm{~A} / 2 \mathrm{~S}$-BS configuration and the smallest change for the $2 \mathrm{~B} / 2 \mathrm{C}-\mathrm{BS}$ configuration.

\subsection{Experimental test setup and procedure}




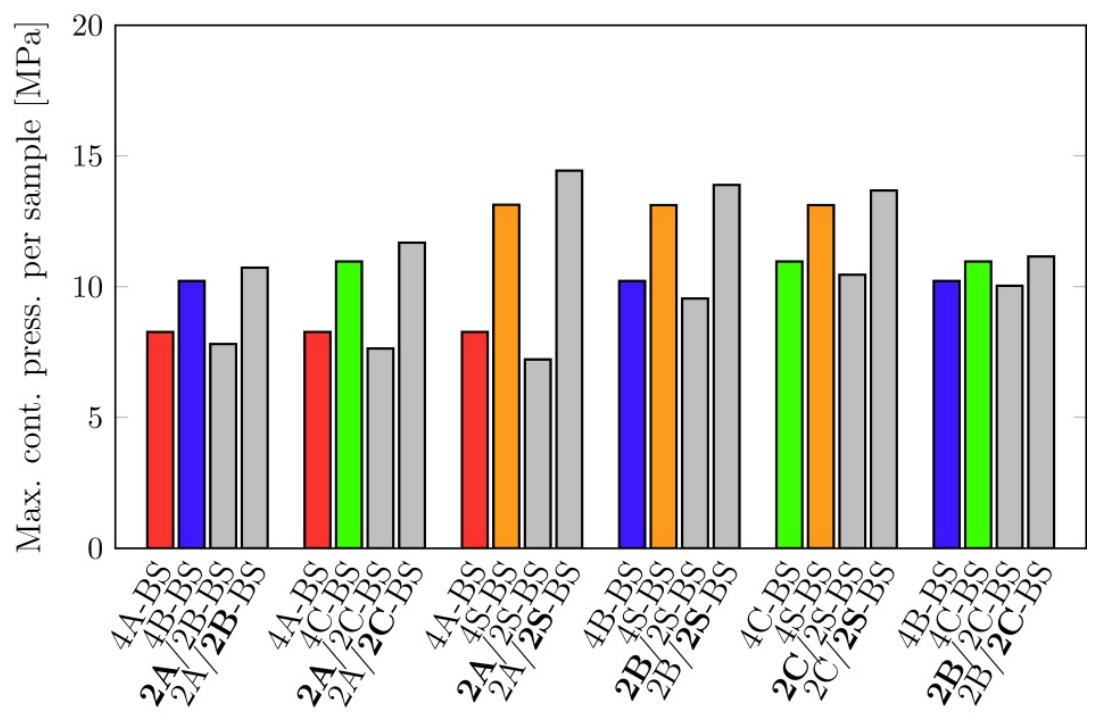

Fig. 3 Maximum contact pressure per sample for the basic and combined configurations (corresponding samples are marked in bold)

A schematic representation of the used test bench, consisting of a rotational tribometer and the ball-on-pyramid test setup, is illustrated in Figure 4. A ball is clamped into a rotating holder, which is

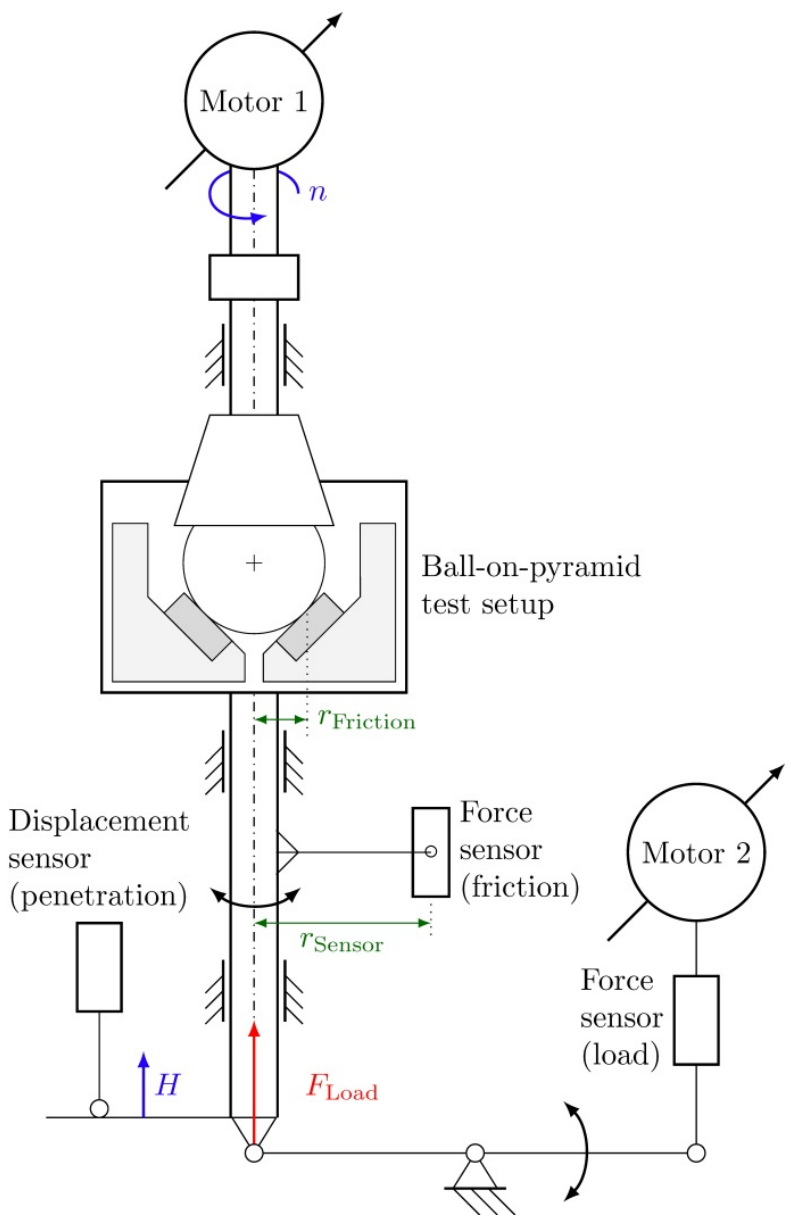

Fig. 4 Schematic representation of the used test bench connected to a servomotor. The pyramid-like sample holder is mounted on a vertically movable platform. The normal load is applied by a motorized spindle and a lever mechanism, which moves the platform in the upward direction. In order to achieve a constant normal load during the tests, a force feedback controller is used. A force sensor, which is installed between a lever connected to the pyramid-like holder and the frame of the test bench, is used to measure the resulting friction force between the rotating ball and the flat samples.

The following relationship was used to obtain the kinetic coefficient of friction:

$$
\mu=\frac{r_{\text {Sensor }} F_{\text {Sensor }}}{r_{\text {Friction }} F_{\mathrm{N}}}=\frac{r_{\text {Sensor }} F_{\text {Sensor }}}{(D / 2) \cos (\alpha) F_{\mathrm{N}}},
$$

with $r_{\text {Friction }}$ denoting the friction radius, $r_{\text {Sensor }}$ the distance between the rotational axis and the force sensor, $F_{\mathrm{N}}$ the normal load, and $F_{\text {Sensor }}$ the measured friction force at the force sensor. The coefficient of friction reached a roughly constant level after an initial running-in period as can be seen in Figure 5 for two typical coefficient of friction curves. Therefore, the average value of the last two-thirds of the total sliding distance was considered for evaluation.

Using a displacement sensor, the vertical displacement of the sample holder towards the rotating ball holder was measured. Because both the flat samples and the bearing steel balls wear out, the penetration depth was considered instead of the volume loss for evaluation. In Figure 2, the penetration depth $h$ of the rotating ball into the flat samples due to wear is illustrated. The relation between the measured vertical displacement $H$ and the penetration depth is

$$
h=\cos (\alpha) H \text {. }
$$

All wear curves showed an approximately linear evolution after an initial running-in phase. Figure 6 illustrates two typical curves for the evolution of the 


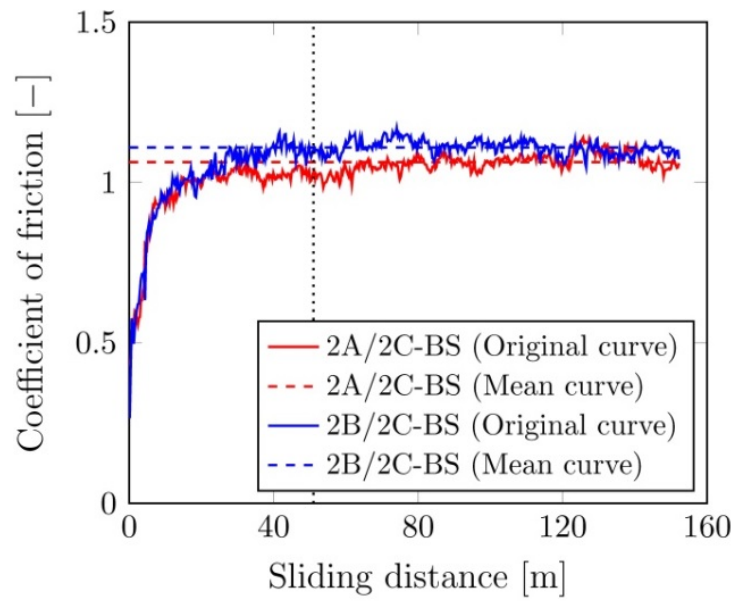

Fig. 5 Exemplary coefficient of friction curves and the corresponding mean curves for the $2 \mathrm{~A} / 2 \mathrm{C}-\mathrm{BS}$ and the $2 \mathrm{~B} / 2 \mathrm{C}-\mathrm{BS}$ configurations

penetration depth. The linear part was approximated by the following linear function:

$$
h_{\text {Linear }}(s)=h_{0}+w_{\mathrm{h}, \mathrm{s}} s \text {, }
$$

with $h_{0}$ depicting the running-in wear and $w_{\mathrm{h}, \mathrm{s}}$ the slope of the curve, respectively the linear wear with regard to the sliding distance. Because both the flat samples and the bearing steel balls experience wear, the linear wear rate describes the combined wear behavior. The linear fitting was applied to the last two-thirds of the total sliding distance.

The stepwise evolution of the penetration depth curves results from the resolution of the displacement sensor. Since linear fitting is applied, this stepwise evolution has no effect on the evaluation of the wear characteristics (e. g., initial wear and linear wear rate).

The wear scars on the flat samples were examined with a microscope after the tests. The scar shapes ranged from circular to elliptical forms. Therefore, a total average equivalent diameter was estimated for a qualitative comparison.

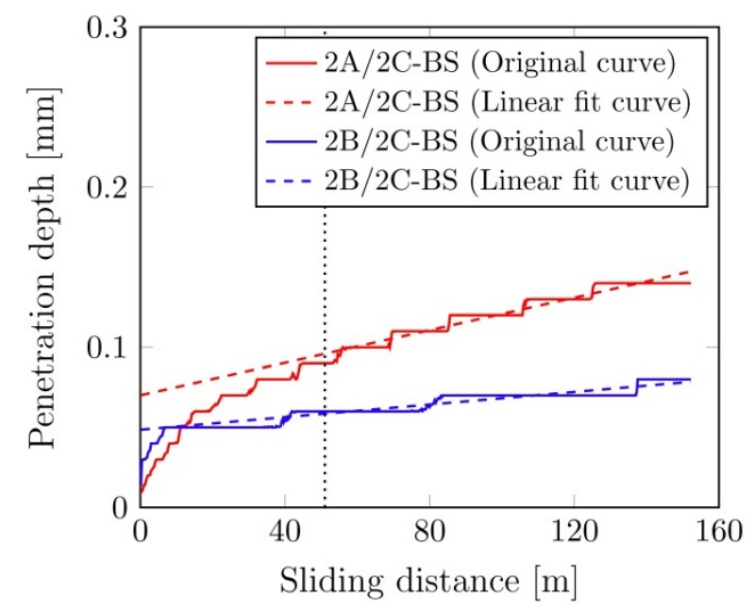

Fig. 6 Exemplary penetration depth and linear fit curves for the $2 \mathrm{~A} / 2 \mathrm{C}-\mathrm{BS}$ and the $2 \mathrm{~B} / 2 \mathrm{C}-\mathrm{BS}$ configurations
The weight loss of the flat samples was usually less than $1 \mathrm{mg}$. Therefore, only the average weight loss of the bearing steel balls was obtained after the tests. But no wear rate was calculated because no linear wear behavior can be expected.

For all tests, the total sliding distance was $s=153 \mathrm{~m}$. The tests were conducted under dry conditions at a room temperature of $21^{\circ} \mathrm{C}$ and a relative humidity of $20 \%$.

The tests were repeated at least three times for every contact configuration. All presented results in this contribution are the arithmetic mean values of the measured values, unless otherwise stated. The corresponding standard deviation is indicated by error bars, describing the scatter of the results.

\section{Results and discussion}

\subsection{Friction behavior}

In Figure 7, the average coefficient of friction for the basic and the combined contact configurations is presented. For a better illustration and comparison, the results for the basic and the combined contact configurations are grouped.

For the 2A/2B-BS configuration, a higher coefficient of friction compared to the corresponding values of the basic configurations can be observed. The coefficient of friction for the $2 \mathrm{~A} / 2 \mathrm{C}$-BS configuration is on the same level as for the basic 4C-BS configuration and higher compared to the basic 4A-BS configuration. The $2 \mathrm{~B} / 2 \mathrm{~S}-\mathrm{BS}$ and the $2 \mathrm{~B} / 2 \mathrm{C}-\mathrm{BS}$ configurations reveal a similar trend. For the combined configurations, the coefficient of friction is higher compared to the basic 4B-BS configuration and slightly smaller compared to the basic 4C-BS, respectively the 4S-BS configuration. For the $2 \mathrm{~A} / 2 \mathrm{~S}-\mathrm{BS}$ and the $2 \mathrm{C} / 2 \mathrm{~S}-\mathrm{BS}$ configurations, the case is different. The coefficient of friction for the combined configurations is below the levels of the basic configurations.

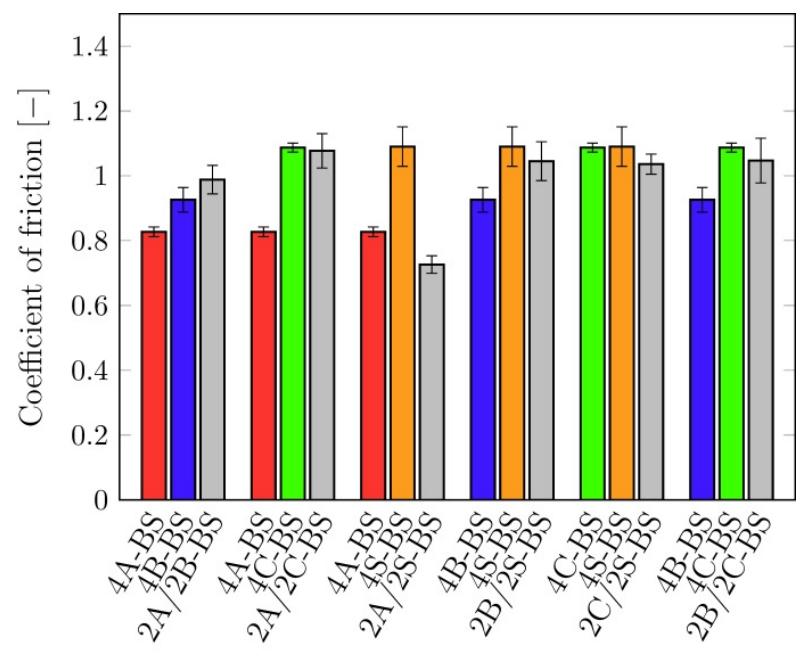

Fig. 7 Average coefficient of friction for the basic and the combined configurations 
The friction characteristics of the combined configurations involving $2 \mathrm{C}$ samples are dominated by the behavior of this samples as the comparison with the values of the basic 4C-BS configuration suggests. The coefficients of friction for the combined configurations involving $2 \mathrm{~S}$ samples are smaller compared to the basic 4S-BS configuration, indicating a less dominating character of this samples. The $2 \mathrm{~A}$ and $2 \mathrm{~B}$ samples in combined configurations seem to have a lesser impact compared to the other sample materials. An exception can be observed for the $2 \mathrm{~A} / 2 \mathrm{~S}-\mathrm{BS}$ configuration where the coefficient of friction of the combined configuration is below the level of both basic configurations.

\subsection{Wear behavior}

Figures 8 and 9 illustrate the average running-in wear and the linear wear rate for the basic and the combined configurations.

The running-in wear for the $2 \mathrm{~A} / 2 \mathrm{C}-\mathrm{BS}$, the $2 \mathrm{~B} / 2 \mathrm{~S}-\mathrm{BS}$, and the $2 \mathrm{C} / 2 \mathrm{~S}-\mathrm{BS}$ configurations is smaller compared to the corresponding basic configurations. The values for the $2 \mathrm{~A} / 2 \mathrm{~B}-\mathrm{BS}$ and $2 \mathrm{~B} / 2 \mathrm{C}-\mathrm{BS}$ configurations are between the values of the corresponding basic pairings. Only for the $2 \mathrm{~A} / 2 \mathrm{~S}-\mathrm{BS}$ configuration, the running-in wear is higher compared to the corresponding basic pairings. A dominating influence of a certain sample material is not evident.

The linear wear rates reveal a different picture. The $2 \mathrm{C}$ and $2 \mathrm{~S}$ samples seem to have a prevailing character in combined configurations (like the $2 \mathrm{~A} / 2 \mathrm{C}-\mathrm{BS}$, the $2 \mathrm{~B} / 2 \mathrm{~S}-\mathrm{BS}$, the $2 \mathrm{C} / 2 \mathrm{~S}-\mathrm{BS}$, and the $2 \mathrm{~B} / 2 \mathrm{C}-\mathrm{BS}$ configuration). The wear rates are usually on the level of the basic configurations involving these samples. Only for the 2A/2B-BS configuration, a smaller and for the $2 \mathrm{~A} / 2 \mathrm{~S}-\mathrm{BS}$ configuration, a higher linear wear rate compared to the corresponding values of the basic configurations can be observed.

The average and individual diameters of the wear scars on the flat samples are shown in Figure 10.

Besides minor deviations, the wear scar sizes are in good agreement with the linear wear rates. Only the scar diameter for the basic 4C-BS configuration is considerably larger in relation to the diameters of the other basic pairings. The wear scar diameters for the combined 2A/2C-BS, 2C/2S-BS, and 2B/2C-BS configurations are smaller compared to the basic configurations.

The individual wear scar diameters (marked in bold) indicate how the single flat samples of the combined contact configurations wear out. The $2 \mathrm{~A}$ and the $2 \mathrm{~S}$ samples experience in all corresponding combined contact configurations the highest wear in comparison to the other sample materials. For configurations involving $2 \mathrm{C}$ and $2 \mathrm{~B}$ samples, the case is vice versa except for the case where both sample materials are involved.

The average weight loss of the bearing steel balls is given in Figure 11.

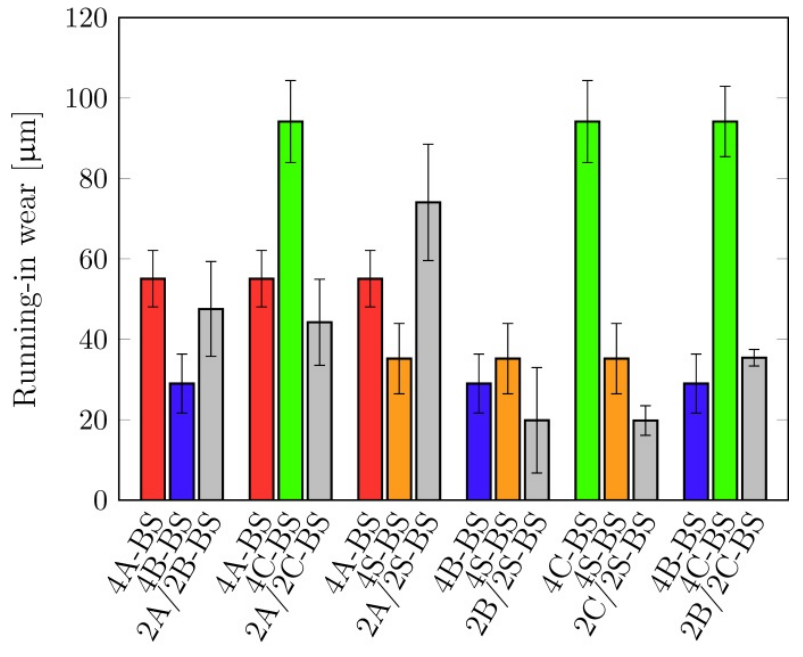

Fig. 8 Average running-in wear for the basic and the combined configurations

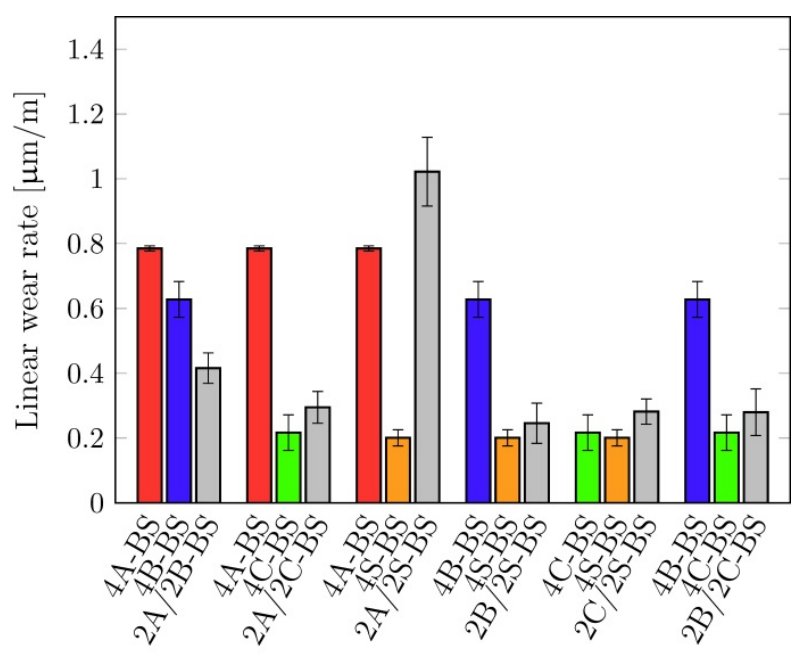

Fig. 9 Average linear wear rate for the basic and the combined configurations

The weight loss for the $2 \mathrm{~A} / 2 \mathrm{~B}-\mathrm{BS}$ and the $2 \mathrm{C} / 2 \mathrm{~S}-\mathrm{BS}$ configurations is between the values of the corresponding basic configurations. For the $2 \mathrm{~A} / 2 \mathrm{~S}-\mathrm{BS}$ and the $2 \mathrm{~B} / 2 \mathrm{C}$ - $\mathrm{BS}$ configurations, the weight loss is lower with regard to the basic configurations. The values for the $2 \mathrm{~B} / 2 \mathrm{~S}-\mathrm{BS}$ configuration are smaller compared to the values for the basic 4B-BS configuration and are in the range of the 4S-BS configuration. The $2 \mathrm{~A} / 2 \mathrm{C}-\mathrm{BS}$ configuration shows a similar behavior in relation to the basic pairings. The lowest weight loss can be observed for the combined 2A/2S-BS configuration.

\subsection{Summary}

A summary of a qualitative comparison of the combined configurations with respect to the basic configurations regarding the coefficient of friction $(\mu)$, the running-in wear $\left(h_{0}\right)$, the linear wear rate $(\dot{w})$, the wear scar diameter on the flat samples $\left(d_{\mathrm{w}}\right)$, and the 


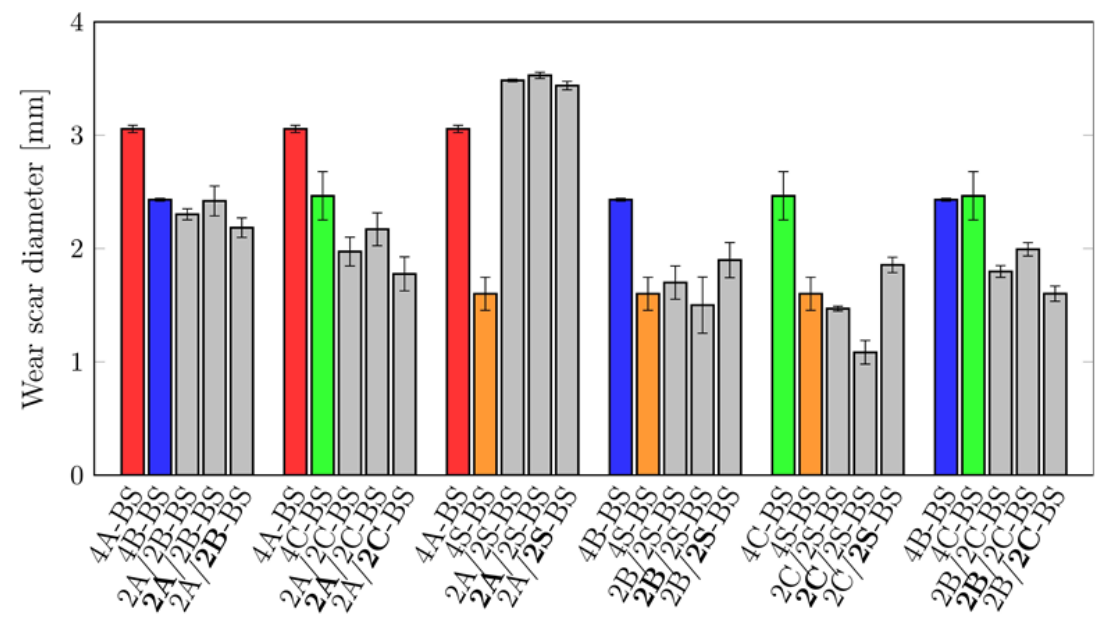

Fig. 10 Average and individual (corresponding samples are marked in bold) wear scar diameters on the flat samples for the basic and the combined configurations

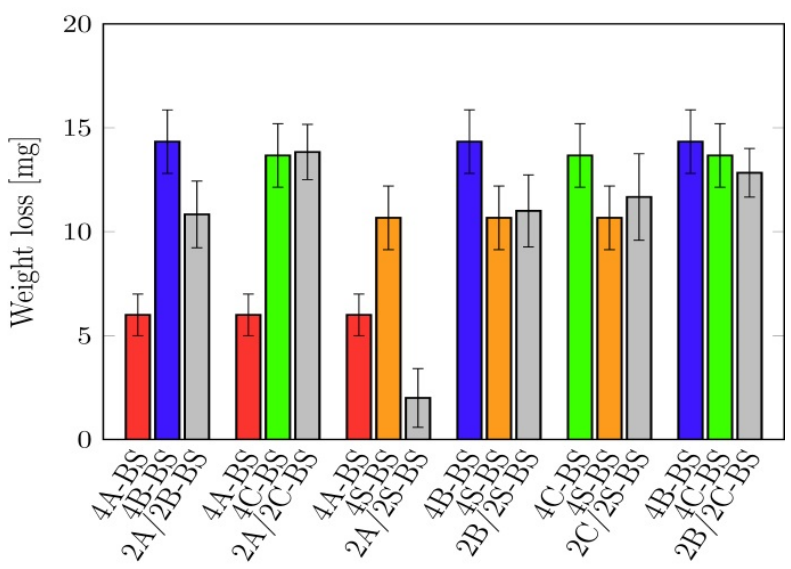

Fig. 11 Average weight loss of the steel balls for the basic and the combined configurations

weight loss $(\Delta m)$ of the bearing steel balls is given in Table 3.

A decrease of the friction force, respectively the coefficient of friction can only be observed for the $2 \mathrm{~A} / 2 \mathrm{~S}-\mathrm{BS}$ and the $2 \mathrm{C} / 2 \mathrm{~S}-\mathrm{BS}$ configurations, whereas for the other combined configurations, the coefficient of friction increases or is in the range of the basic configuration with the higher coefficient of friction.

In principle, the $2 \mathrm{~A} / 2 \mathrm{~B}-\mathrm{BS}$, the $2 \mathrm{~A} / 2 \mathrm{C}-\mathrm{BS}$, the $2 \mathrm{~B} / 2 \mathrm{~S}-\mathrm{BS}$, and the $2 \mathrm{~B} / 2 \mathrm{C}-\mathrm{BS}$ configurations show similar trends regarding the coefficient of friction and the wear scar diameter. The coefficient of friction of the combined configurations is higher or in between the values of these configurations, whereas the wear scar diameters are smaller compared to the basic configurations.

A clear decrease of the linear wear rate compared to the basic configurations is only evident for the 2A/2B-BS configuration.

Even though wear debris could escape from the contact zones due to the obliquely arranged samples, for the combined $2 \mathrm{~A} / 2 \mathrm{~S}-\mathrm{BS}$ configuration it was observed that some debris was still trapped and adhered to the roughened surface. Additionally, the corresponding low weight loss of the bearing steel balls and the comparatively high running-in wear, linear wear rate, and wear scar diameter indicate a material transfer from the flat samples to the steel ball and therefore confirm that observation. This mechanism was noticed only for the $2 \mathrm{~A} / 2 \mathrm{~S}-\mathrm{BS}$ configuration. In Figure 12 the wear marks on the bearing steel balls resulting from a 2B/2S-BS configuration (without any wear debris adhered to the surface) and from a $2 \mathrm{~A} / 2 \mathrm{~S}-\mathrm{BS}$ configuration (with wear debris adhered to the surface) are compared.

Abrasive wear can be divided into sliding abrasion (two-body wear) and rolling abrasion (three-body wear). Sliding abrasion describes material removal from the softer surface by asperities of the harder surface or by hard particles attached to one of the sliding surfaces. In the case of rolling abrasion, free particles are trapped/involved between and interact with the sliding surfaces. The contact configuration, the loading condition, the generation of wear particles, the mechanical properties of the particles, and whether the particles remain fully or partly in the contact zone determine the actual wear mechanisms.

In the present case, sliding abrasion was predominant. However, it cannot completely be ruled out that some wear debris remained in the contact zone. Therefore, rolling abrasion is also possible to some extent. An important role in this context plays the hardness (see Table 1) of the samples and of the generated wear particles regarding the abrasive wear resistance. While the comparatively hard bearing steel balls slide over the flat samples, it can be expected that the softer samples experience more abrasive wear than the harder samples. The observed weight loss of the bearing steel balls indicates that the steel balls also wear out during the test due to abrasive wear. 
Table 3 Qualitative comparison of the combined configurations with respect to the basic configurations

\begin{tabular}{c|c|c|c|c|c|c}
$\begin{array}{c}\text { Combined } \\
\text { configuration }\end{array}$ & $\begin{array}{c}\text { Basic } \\
\text { configuration }\end{array}$ & $\boldsymbol{\mu}$ & $\boldsymbol{h}_{\mathbf{0}}$ & $\dot{\boldsymbol{w}}$ & $\boldsymbol{d}_{\mathbf{w}}$ & $\boldsymbol{\Delta} \boldsymbol{m}$ \\
\hline \multirow{2}{*}{$2 \mathrm{~A} / 2 \mathrm{~B}-\mathrm{BS}$} & $4 \mathrm{~A}-\mathrm{BS}$ & $\uparrow$ & $\downarrow$ & $\downarrow$ & $\downarrow$ & $\uparrow$ \\
\cline { 2 - 7 } & $4 \mathrm{~B}-\mathrm{BS}$ & $\uparrow$ & $\uparrow$ & $\downarrow$ & $\downarrow$ & $\downarrow$ \\
\hline \multirow{2}{*}{ 2A/2C-BS } & $4 \mathrm{~A}-\mathrm{BS}$ & $\uparrow$ & $\downarrow$ & $\downarrow$ & $\downarrow$ & $\uparrow$ \\
\cline { 2 - 7 } & $4 \mathrm{C}-\mathrm{BS}$ & $\approx$ & $\downarrow$ & $\uparrow$ & $\downarrow$ & $\approx$ \\
\hline \multirow{2}{*}{$2 \mathrm{~A} / 2 \mathrm{~S}-\mathrm{BS}$} & $4 \mathrm{~A}-\mathrm{BS}$ & $\downarrow$ & $\uparrow$ & $\uparrow$ & $\uparrow$ & $\downarrow$ \\
\cline { 2 - 7 } & $4 \mathrm{~S}-\mathrm{BS}$ & $\downarrow$ & $\uparrow$ & $\uparrow$ & $\uparrow$ & $\downarrow$ \\
\hline \multirow{2}{*}{ 2B/2S-BS } & $4 \mathrm{~B}-\mathrm{BS}$ & $\uparrow$ & $\downarrow$ & $\downarrow$ & $\downarrow$ & $\downarrow$ \\
\cline { 2 - 7 } & $4 \mathrm{~S}-\mathrm{BS}$ & $\downarrow$ & $\downarrow$ & $\uparrow$ & $\approx$ & $\approx$ \\
\hline \multirow{2}{*}{$2 \mathrm{C} / 2 \mathrm{~S}-\mathrm{BS}$} & $4 \mathrm{C}-\mathrm{BS}$ & $\downarrow$ & $\downarrow$ & $\uparrow$ & $\downarrow$ & $\downarrow$ \\
\cline { 2 - 7 } & $4 \mathrm{~S}-\mathrm{BS}$ & $\downarrow$ & $\downarrow$ & $\uparrow$ & $\downarrow$ & $\uparrow$ \\
\hline \multirow{2}{*}{ 2B/2C-BS } & $4 \mathrm{~B}-\mathrm{BS}$ & $\uparrow$ & $\uparrow$ & $\downarrow$ & $\downarrow$ & $\downarrow$ \\
\cline { 2 - 7 } & $4 \mathrm{C}-\mathrm{BS}$ & $\downarrow$ & $\downarrow$ & $\uparrow$ & $\downarrow$ & $\downarrow$
\end{tabular}

Among the basic configurations, the 4A-BS configuration shows the highest linear wear rate and the lowest weight loss of the steel balls. For the 4B-BS configuration, the case is vice versa. Since the hardness of the 4B samples is higher compared to the 4A samples, the weight loss of the steel balls of the 4B-BS configuration is higher, while the linear wear rate is lower in comparison to the 4A-BS configuration. Nevertheless, this relation does not apply to all configurations as the results involving $4 \mathrm{C}$ samples

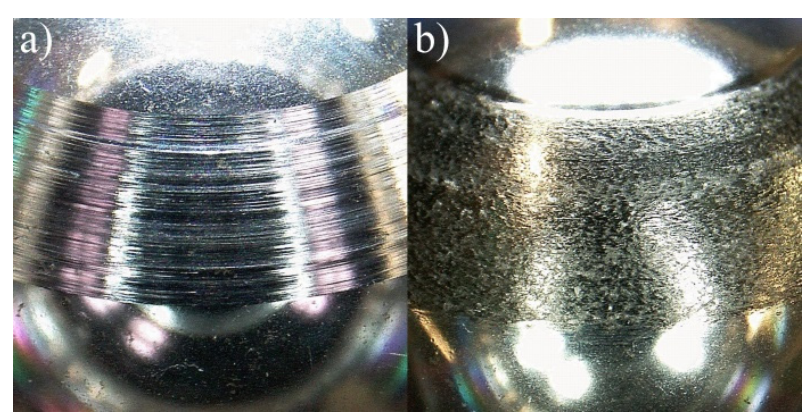

Fig. 12 Wear marks on the bearing steel balls: a) 2B/2S-BS configuration (without any wear debris adhered to the surface), b) $2 \mathrm{~A} / 2 \mathrm{~S}-\mathrm{BS}$ configuration (with wear debris adhered to the surface) suggest. Furthermore, the hardness of the brass and copper samples is in the same range, but the results regarding the wear characteristics for the combined contact configurations involving these samples are different. This is why the observed trends cannot simply be related to the hardness of the samples. In the case of the $2 \mathrm{~A} / 2 \mathrm{~S}-\mathrm{BS}$ configuration, the hardness of the bearing steel ball and the flat steel samples is higher compared to the aluminum samples. The aluminum samples are much softer and therefore experience more wear. In addition, the soft wear particles probably also act as a lubricant to some extent.

Moreover, abrasive wear causes some surface roughening at the contact zones, which in return influences the ongoing wear processes. While the steel ball slides over the soft samples, some particles may attach to its surface and then be removed again when sliding over the hard samples.

This process can be considered as a continuous cycle where the single mechanisms interact and influence each other at the same time. The wear mechanisms and their extent/contribution depend on the mechanical properties of the materials. So, a manipulation of the mechanisms can probably be achieved by using materials with certain mechanical properties.

Relating the results of the friction and wear behavior 
to the contact pressures (see Figure 3) indicates no clear trends. With regard to the contact pressure, a noticeable effect on the wear behavior can be expected for combined configurations involving $2 \mathrm{~S}$ samples, especially for the $2 \mathrm{~A} / 2 \mathrm{~S}-\mathrm{BS}$ configuration. The corresponding results for the running-in wear, the linear wear rate, and the wear scar diameter confirm this expectation mainly only for the $2 \mathrm{~A} / 2 \mathrm{~S}-\mathrm{BS}$ configuration. On the other hand, for the $2 \mathrm{~B} / 2 \mathrm{C}$-BS configuration, the contact pressures do not change substantially, but some noticeable changes in the wear behavior can be observed.

The friction and wear characteristics reveal that the combined configurations are not simply the arithmetic mean values of the basic configurations. The relation and interaction between the different friction and wear mechanisms seems to be much more complex and therefore leads to the observed trends.

Generally, it can be stated that by combining different material pairings, a greater effect on the wear than on the friction behavior can be observed for the studied materials in the investigated range.

The results for the running-in wear and the weight loss of the steel balls exhibit the highest scatter in comparison to the other values. Possible reasons for that could be the vulnerability of the running-in wear to initial elastic and plastic setting processes and the material transfer from the flat samples to the roughened steel balls.

\section{Conclusion and outlook}

The adapted ball-on-pyramid test setup extends the so far existing test procedures and allows to study combined contact configurations consisting of more than one material pairing.

A comparison of the results reveals the potential of some combined contact configurations regarding the friction and/or wear performance. By combining certain materials, a reduction of friction and/or wear is generally possible. Based on the qualitative comparison, the $2 \mathrm{C} / 2 \mathrm{~S}-\mathrm{BS}$ configuration, for example, seems to be the most promising in the context of low friction and low wear.

A common approach to improve the friction and wear performance of tribological systems is the use of appropriate coatings or lubricants. The proposed approach represents a possible alternative to the conventional methods in this context. Whether the observed results can be transferred to real life applications cannot be answered at this point. However, simple sliding systems, like bearing bushings, ball joints, sliding joints, or sliding guides could be a possible field of application.

In further studies, the introduced test setup shall also be used to investigate lubricated contact configurations and different sample materials, like metals or polymers, as well as the combination of both.

\section{References}

[1] Czichos, H., Saito, T. and Smith, L., "Springer Handbook of Materials Measurement Methods," Springer, Berlin, 2006, 295-310.

[2] Blau, P. J., "Friction Science and Technology: From Concepts to Applications," Second Edition, CRC Press, New York, 2008, 44-46.

[3] ASTM D4172-94, 2010.

[4] ASTM D5183-08, 2011.

[5] Voitik, R. M., "Four-Ball Machine with New BOTS Test Permits Diesel Fuel Lubricity Evaluation," SAE ISO/TC22/SC7/WG6-Diesel Fuel Lubricity, Document N127, February 1993, 1-19.

[6] Voitik, R. M. and Ren, N., "Diesel Fuel Lubricity by Standard Four Ball Apparatus Utilizing Ball On Three Disks, BOTD," SAE Technical Paper 950247, Society of Automotive Engineers, 1995, 1-8.

[7] Fildes, J. M., Meyers, S. J., Kilaparti, R. and Schlepp, E., "Improved Ball Crater MicroAbrasion Test Based on a Ball on Three Disk Configuration," Wear, 274-275, 2012, 414-422.

[8] Fildes, J. M., Meyers, S. J., Mulligan, C. P. and Kilaparti, R., "Evaluation of the Wear and Abrasion Resistance of Hard Coatings by Ball-On-ThreeDisk Test Methods-A Case Study," Wear, 302, 1-2, 2013, 1040-1049.

[9] PN-83/H-04302, 1983.

[10] Senatorski, J. and Tacikowski, J., "A New Approach to Wear Testing of Case-Hardened Steels," Tribotest Journal, 3, 3, 1997, 307-323.

[11] Senatorski, J., Tacikowski, J. and Liliental, W., "Tribological Properties of Nitrided Layers, as Determined by the Cone-Three-Cylinder Wear Test," Tribology Transactions, 41, 2, 1998, 199-208.

[12] ISO 7148-2, 2012.

[13] Krasmik, V. and Schlattmann, J., "Experimental Investigation of the Friction and Wear Behaviour with an Adapted Ball-On-Prism Test Setup," Tribology in Industry, 37, 3, 2015, 291-298.

[14] Johnson, K. L., “Contact Mechanics," Cambridge University Press, Cambridge, 1985, 90-104 